\title{
\# www.springerprofessional.de
}

Beitrag des Monats

\section{CFOs bleibt zu wenig Zeit für Strategie}

CFOs sollen CEOs als strategische Partner unterstützen. Und dennoch widmen sie sich laut der „Arbeitsmarktstudie 2015“ des Personaldienstleisters Robert Half kaum strategischen Aufgaben: Nur 5,2 Stunden pro Woche wenden die 200 befragten CFOs für die Unterstützung der Unternehmensstrategie und immerhin sechs Stunden für die Einführung und Umsetzung einer Finanzstrategie auf. Die meiste Zeit, im Schnitt 9,2 Stunden, benötigen die CFOs für die Teamführung (23 Pro- zent). Operative Aufgaben wie zum Beispiel das Finanz-Reporting, Buchhaltung und Audits nehmen rund ein Fünftel der Zeit ein. CFOs müssen also den Spagat hinbekommen, einerseits die klassischen Aufgaben zu erfüllen, andererseits auch die strategischen Aufgaben anzunehmen. CFOs brauchen deshalb vor allem eins: ein gutes Zeitmanagement!

$\underline{\imath}$ Lesen Sie den ganzen Beitrag unter www.springerprofessional.de/financebanking.

\section{Weitere meistgeklickte Beiträge}

\section{2. „Fortschrittliche Planungsinstrumente sollten schlank und flexibel sein"}

\section{Unternehmen planen lange und altmodisch}

4. Welche Vorteile eine GmbH \& Co. KG bietet

5. Wie viel verdienen CFOs und Controller 2016?

\section{Das Wissensportal www.springerprofessional.de}

Unser Wissensportal bündelt die Fachgebiete in Wirtschaft und Technik. Im Fachgebiet „Finance + Banking“ finden Sie aktuelle Informationen und weiterführende Literatur für Controller. Dort ist auch das Archiv der „Controlling \& Management Review“ hinterlegt. Alle Lesetipps im Heft, die mit einem 흐 gekennzeichnet sind, sind für Abonnenten frei zugänglich.

\section{\# www.springerprofessional.de}

